\title{
Cepheids in binary systems - new candidates in the Magellanic Clouds
}

\author{
L. Szabados \\ Konkoly Observatory, Budapest, Hungary; email: szabados@konkoly.hu
}

\begin{abstract}
While in our Milky Way galaxy the majority of Cepheids is not solitary, in the Magellanic Clouds only a few binaries have been revealed in lack of systematic spectroscopic studies. A simple numerical parameter, the ratio of the radial velocity and the photometric amplitudes was studied for the Magellanic Cloud Cepheids. A large value of this amplitude ratio is a hint that the Cepheid has a companion. According to this duplicity indicator HV 834 and HV 2864 are possibly members in binary systems.
\end{abstract}

\section{Introduction}

Owing to their role as primary distance indicators, Cepheids were intensely searched for in other galaxies of the Local Group and even beyond. Up to now Cepheids were found in more than 20 external galaxies but no binary system among them has been identified beyond the Magellanic Clouds. In order to get a reliable zero point of the period-luminosity relationship, the brightness contributions of the companion(s) of Cepheids belonging to binary (or multiple) systems have to be removed before converting the apparent magnitude into absolute brightness. For this purpose Cepheids belonging to binary systems have to be identified.

\section{Binary Cepheids in the Magellanic Clouds}

The Magellanic Clouds are fundamental objects for astronomy in several respects. Classical Cepheids belonging to the Clouds are especially important because the period-luminosity relation has been traditionally calibrated by using Cepheids in these nearby galaxies. In view of the fact that the photometric effects of the companions have to be taken into account when determining the luminosities of the Cepheids, and because of the high incidence of duplicity among Galactic Cepheids, it is essential to study binarity of the Magellanic Cloud Cepheids as well. In this respect, however, our knowledge on companions to Cepheids in the neighbouring galaxies is rather incomplete.

Although several thousand Cepheids have been discovered in the two Clouds, binarity has been established only in 7 cases. This is a serious deficiency because binaries among Cepheids are more frequent than solitary Cepheids (Szabados 2003b). In spite of the strong selection bias, there are about 150 known binaries among the less than a thousand Galactic Cepheids (Szabados 2003a). Three long period spectroscopic binary systems have been found from among eleven Cepheids by Imbert et al. (1985): HV 837 and HV 11157 in the SMC, and HV 883 in the LMC. The orbital elements of these binaries were determined in a subsequent paper by Imbert (1994). The fourth single lined spectroscopic binary among Magellanic Cloud Cepheids was discovered during a detailed photometric/spectroscopic study of the LMC cluster NGC 1866 (Welch et al., 1991), but the radial velocity data obtained in two 
consecutive years have been insufficient to derive the orbital period (and other elements) of the system involving the Cepheid HV 12202.

The extensive photometry carried out for finding microlensing events resulted in discovering 3 eclipsing binaries from among nearly 1800 MACHO Cepheids in the Magellanic Clouds (Alcock et al., 2002). Based on their location in the colour-magnitude diagram, MACHO 81.8997.87 is a Cepheid pulsating in the first overtone $\left(P_{\text {puls }}=2.035\right.$ days, $P_{\text {orb }}=800.5$ days $)$, MACHO $6.6454 .5\left(P_{\text {puls }}=4.974\right.$ days, $P_{\text {orb }}=397.142$ days $)$ has a lower luminosity than the fundamental mode pulsators if the flux from the companion is removed properly, while the third Cepheid in an eclipsing binary system, MACHO 78.6338.24 $\left(P_{\text {puls }}=17.686\right.$ days, $P_{\text {orb }}=419.718$ days $)$ is a Population II Cepheid.

\section{Ratio of radial velocity and photometric amplitudes as a hint for duplicity}

The ratio of the radial velocity and photometric amplitudes (determined for the complete pulsational cycle) as a possible indicator of duplicity was first mentioned by Coulson and Caldwell (1989) but in their study this criterion was only applied to a limited sample.

If there existed a normal value of the amplitude ratio $A_{\mathrm{VRAD}} / A_{B}$ for a given pulsation period, it would be expected that in the presence of a companion one observes a modified value: the photometric data result in a decreased amplitude, while the radial velocity variations of pulsational origin are not affected by the companion. Moreover, the unrecognized orbital motion superimposed on the pulsational changes results in an increased $A_{\mathrm{VRAD}}$. Therefore a larger-than-normal value of $A_{\mathrm{VRAD}} / A_{B}$ (in what follows, this amplitude ratio is referred to as $A R$ ) may indicate presence of a companion.

The behaviour of the $A R$ has been studied using the data on more than a hundred classical Cepheids in the Magellanic Clouds and the Milky Way for which reliable radial velocity and photometric data have been available (via the on-line data base compiled by D. L. Welch at the URL: http://dogwood.physics.mcmaster.ca/Cepheid/HomePage.html). The diagram showing $A R$ against the logarithm of the pulsation period is seen in Figure 1. In this plot, circles denote fundamental pulsators, squares mean the $A R$ values for the first overtone mode. The overtone pulsation can be readily identified if the Cepheid is either in the Magellanic Clouds (the overtone pulsators are more luminous in the colour-magnitude diagram), or pulsates in two modes simultaneously (beat Cepheid). A beat Cepheid can be characterized by two $A R$ values, one for each excited mode. Some general conclusions can be deduced from Figure 1:

- At a given pulsation period, no normal value of the $A R$ exists. At any period there is a large spread in both photometric and radial velocity amplitudes;

- The $A R$ value is systematically larger for overtone pulsators than for the fundamental mode;

- For Cepheids pulsating in the fundamental mode, the average $A R$ is independent of the pulsation period itself.

The larger $A R$ of the overtone pulsators is a consequence of the higher frequency of the overtone as compared with the fundamental mode oscillation of the same star. From their linear pulsational model Balona and Stobie (1979) derived that

$$
A_{V_{\mathrm{RAD}}} / A_{V}=4.097 R_{0} /\left[P\left(f^{2}+4 f \cos \psi+4\right)^{1 / 2}\right]
$$

where $R_{0}$ is the average radius, $\psi$ is the phase lag between the moments of the maximum flux and maximum radius, $f$ is the ratio of flux variation to radius variation. Because the period ratio of the two excited modes is about 0.7 for beat Cepheids, one expects $1 / 0.7$ times larger value of $A R$ for the first overtone pulsation as compared with the fundamental mode oscillation (considering a given photometric band). The numerical values of the $A R$ determined from the observational data of Galactic double-mode Cepheids are in accordance with this theoretical value. 


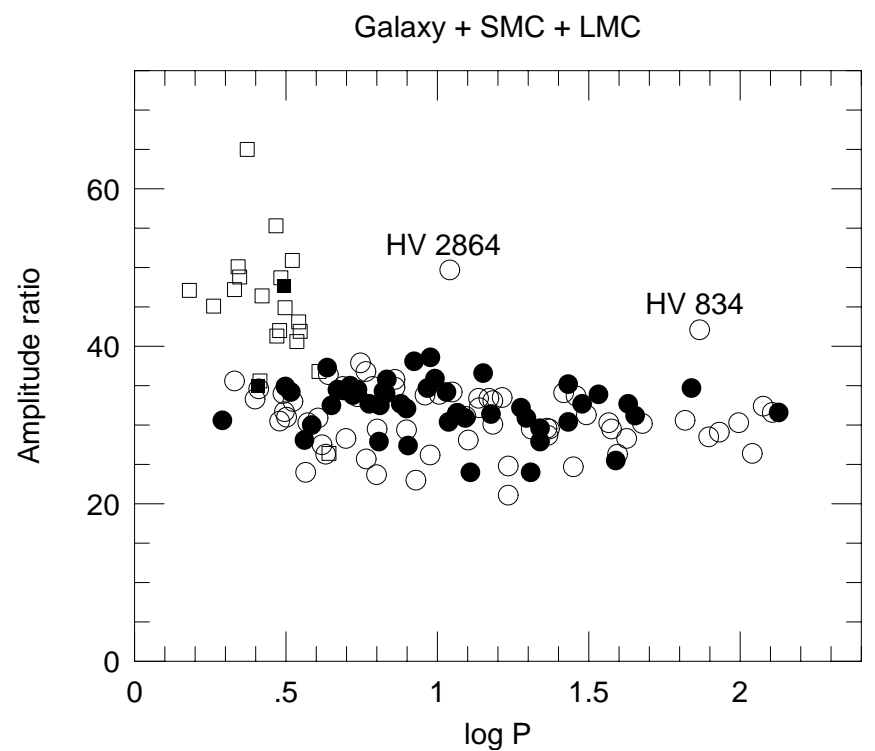

Figure 1: The $A R$ (defined in the text) as a function of the pulsation period. Circles denote $A R$ values of fundamental mode pulsators, squares are used for denoting Cepheid oscillating in the first overtone. Binaries, marked with filled symbols, tend to have larger values of the amplitude ratio than solitary Cepheids. The two most upwardly deviating stars, HV 2864 and HV 834, are newly suspected binaries.

In order to demonstrate the effect of binarity on the $A R$ in Figure 1, Cepheids with known companions are marked with filled symbols, while empty symbols denote solitary Cepheids as well as those stars, whose companions have not been detected yet. It is seen that, in accordance with the expectation, binaries tend to have larger $A R$ values on average than solitary Cepheids. Keeping in mind, however, the width of the interval of normal $A R$ values, the larger-than-average value of the $A R$ does not necessarily imply duplicity of the individual variables. There are, however, two Cepheids, HV 834 in the SMC and HV 2864 in the LMC whose extremely large amplitude ratio may well be caused by a companion star.

Acknowledgments. The author expresses his gratitude to Dr. Mária Kun for her useful comments on the manuscript. Financial support from the Hungarian OTKA grant T34584 is acknowledged.

\section{References}

Alcock C., Allsman R. A., Alves, D. R., et al. (The MACHO Collaboration), 2002, ApJ 573, 338

Balona L. A., Stobie R. S., 1979, MNRAS 189, 649

Coulson I. M., Caldwell J. A. R., 1989, MNRAS 240, 285

Imbert M., 1996, A\&AS 116, 497

Imbert M., Andersen J., Ardeberg A., et al., 1985, A\&AS 81, 339

Szabados L., 2003a, Inf. Bull. Var. Stars, No. 5394

Szabados L., 2003b, in GAIA Spectroscopy, Science and Technology, ed. U. Munari, ASP Conf. Ser. 298 (San Francisco: ASP), p. 237

Welch D. L., Mateo M., Coté P., Fischer P., Madore B. F., 1991, AJ 101, 490 